\title{
CULTIVO HIDROPÓNICO DE ESPINACA MEDIANTE TÉCNICA NFT E INVERNADERO PARA EL CONTROL DE VARIABLES AMBIENTALES
}

\author{
Hydroponics culture of spinach through NFT technique \\ and greenhouse to control environmental variables
}

\author{
Diego Chiara, Luis Herrera, Paolo Vargas ${ }^{1}$ \\ Adviser Mario Chauca ${ }^{2}$
}

PRESENTACIÓN: MAYO 2016

ACEPTACIÓN: JULIO 2016

\section{RESUMEN}

El presente trabajo expone la elaboración de un producto hidropónico combinando la técnica de irrigación con nutrientes (NFT) con el aprovechamiento del espacio, cuidado y condiciones ambientales de invernadero (temperatura, humedad e iluminación). Los controles electrónicos automatizados hacen el proyecto más valioso, ya que permiten el cuidado óptimo del cultivo con ahorro de energía, tiempo y trabajo manual. El control total de un producto alimenticio es beneficioso desde el punto de vista de un negocio que comercializa comida saludable y de calidad.

Palabras clave: NFT, invernadero, hortalizas, sensores, Arduino, hidroponía, ahorro de insumos

\begin{abstract}
Combining the NFT technique for irrigation with nutrients utilizing the space and gravity, caring and greenhouse environmental conditions (temperature, humidity and light) conducive to hydroponics product is made. Automation system electronically makes the project as enriching thus allow optimum control with saving energy, time and hand work. The total control of a food product would be beneficial from the point of view of a productive business to support healthy and quality food.
\end{abstract}

Keywords: NFT, greenhouse, vegetables, sensors, Arduino, hydroponics, supplies savings. 


\section{NOMENCLATURA}

NFT: Nutrient film technique

\section{INTRODUCCIÓN}

La hidroponía es una técnica que sirve para cultivar plantas usando soluciones minerales sin suelo agrícola. Las raíces reciben una solución nutritiva equilibrada disuelta en agua con todos los elementos esenciales para el crecimiento de la planta. Este método es útil para cultivar verduras y vegetales ricos en vitaminas y minerales de una manera limpia y sana (Castañeda, 1997, pp. 1-6).

La espinaca (Spinacia oleracea L.) es un cultivo muy extendido a nivel mundial, ampliamente utilizado en la industria alimenticia. En los últimos ańos, se viene produciendo en forma de baby leaf para su comercialización como producto mínimamente procesado. El sistema de cultivo en bandejas flotantes es una técnica sencilla para la producción de hortalizas de pequeño tamaño entre ellas, verduras de hoja pequeña tipo baby leaf. Este tipo de cultivo es un complemento ideal para la agricultura tradicional. Las raíces de las plantas reciben una solución nutritiva equilibrada disuelta en agua con todos los elementos químicos imprescindibles para el desarrollo de la planta. Por ser un tipo de cultivo limpio, ecológico, renovable y muy eficiente, con esta técnica se contribuye al equilibrio del ambiente en el hogar (Murray, 1993). Actualmente, en el Perú, los productos que se cultivan no suelen cumplir con estándares alimenticios, pues no son totalmente naturales y su producción es para fines lucrativos y no para el buen consumo humano. En esta coyuntura, los cultivos hidropónicos ofrecen productos de mayor calidad, es decir, libres de malezas y con una solución nutritiva balanceada y equilibrada (Via, 2009).

El objetivo general de este proyecto es diseñar un sistema de control para un cultivo hidropónico. En ese sentido, el NFT nos permite reciclar el agua con nutrientes y, mediante una bomba eléctrica, hacerla discurrir por todos los sectores donde se ubica el producto (Sampero, 1999a). El invernadero que cubre el contenedor dotará al sistema de un ambiente propicio para el crecimiento de la planta. Por su parte, los sensores de temperatura y humedad controlarán directamente las variables físicas que serán displayadas para su interpretación (Daraviña, 2014). A través de este proyecto, se pretende comprender todo lo relacionado con el diseño de un control ambiental con la intención de ofrecer un modelo para un sistema hidropónico propio, ya sea escolar, familiar o comunitario con el cual se pueda obtener, en poco tiempo, verduras sanas y frescas para el consumo. Asimismo, se busca aportar soluciones adecuadas para las necesidades del sector agroindustrial del país (Castañeda, 1997).

TABLA I. CUADRO DESCRIPTIVO DE NUTRIENTES DE HORTALIZAS

\begin{tabular}{lc}
\hline \multicolumn{1}{c}{ Fuente } & g y ml por cada 1000L de solución a preparar \\
\hline 1. Ácido fosfórico (85\%) & $87.5 \mathrm{ml}^{*}$ \\
\hline 2. Nitrato de calcio (15.5\%N, 19\%Ca) & $574 \mathrm{~g}$ \\
\hline 3. Nitrato de potasio (12\%N, 46\%K) & $375 \mathrm{~g}$ \\
\hline 4. Sulfato de magnesio (10\% Mg) & $410 \mathrm{~g}$ \\
\hline 5. Sulfato de potasio (44.5\%K) & $125 \mathrm{~g}$ \\
\hline 6. Multi micro comb (Haifa)(Fe 7.5\%, Mn 3.5\%, Zn & $18.5 \mathrm{~g}$ \\
0.7\%, B 0.65\%, Cu 0.28\%, Mo 0.26\%) & \\
\hline
\end{tabular}


Las hortalizas cumplen con el factor tiempo de crecimiento. La espinaca como vegetal es altamente nutritiva y, por ende, es consumida masivamente por el hombre (Alpízar, 2004).

TABLA II. VENTAJA NUTRICIONAL DE LA ESPINACA CON RESPECTO A OTRAS HORTALIZAS

\begin{tabular}{|c|c|c|c|c|c|c|c|c|c|c|c|}
\hline Hortaliza & Cal $100 \mathrm{~g}$ & $\mathrm{Na} \mathrm{mg}$ & Ca mg & Fe mg & P mg & $\mathrm{Kmg}$ & V.A U.I. & V. B1 mg & V. B2 mg & $\begin{array}{c}\text { V. BE } \\
m g\end{array}$ & $\begin{array}{l}\text { V.C } \\
\text { mg }\end{array}$ \\
\hline Acelga & 25 & 140 & 90 & 3.5 & 39 & 400 & 6500 & 0.04 & 0.15 & 0.5 & 34 \\
\hline Apio & 18 & 115 & 30 & 0.5 & 30 & 800 & - & 0.03 & 0.03 & 0.3 & 9 \\
\hline Berenjena & 25 & 2 & 15 & 0.5 & 30 & 210 & 10 & 0.05 & 0.05 & 0.6 & 5 \\
\hline Berro & 20 & 50 & 150 & 1.9 & 60 & 282 & 4900 & 0.1 & 0.15 & 0.9 & 75 \\
\hline Brócoli & 35 & 16 & 105 & 1.3 & 78 & 400 & 2500 & 0.1 & 0.15 & 0.9 & 100 \\
\hline Coliflor & 28 & 18 & 27 & 1 & 56 & 300 & 60 & 0.11 & 0.11 & 0.7 & 75 \\
\hline Esparrago & 24 & 4 & 24 & 1 & 50 & 240 & 855 & 0.12 & 0.12 & 1.4 & 25 \\
\hline Espinaca & 28 & 70 & 80 & 3.5 & 50 & 500 & 5000 & 0.1 & 0.2 & 0.6 & 45 \\
\hline Hinojo & 25 & 86 & 100 & 2.5 & 55 & 400 & 3500 & 0.23 & 0.11 & 0.2 & 93 \\
\hline Lechuga & 13 & 9 & 20 & 0.5 & 23 & 175 & 330 & 0.05 & 0.05 & 0.3 & 7 \\
\hline Pepino & 15 & 5 & 20 & 0.8 & 23 & 170 & 250 & 0.03 & 0.04 & 0.2 & 13 \\
\hline Rabanito & 15 & 17 & 20 & 1.2 & 35 & 320 & 10 & 0.03 & 0.03 & 0.3 & 26 \\
\hline $\begin{array}{l}\text { Col de } \\
\text { Bruselas }\end{array}$ & 40 & 11 & 22 & 1.5 & 80 & 400 & 550 & 0.11 & 0.14 & 0.9 & 100 \\
\hline Tomate & 21 & 3 & 12 & 0.5 & 26 & 240 & 900 & 0.06 & 0.04 & 0.7 & 23 \\
\hline
\end{tabular}

TABLA III. CUADRO COMPARATIVO DE SUELO NORMAL Y SUSTRATO HIDROPÓNICO

\begin{tabular}{lcc}
\hline \multicolumn{1}{c}{ CARACTERÍsTICAS } & SUELO & SUSTRATO \\
\hline Material sólido & $50 \%$ & $15 \%$ \\
\hline Espacio poroso total & $50 \%$ & $85 \%$ \\
\hline Densidad & Pesados $\mathrm{LL}=1500 \mathrm{gr}$ & Ligeros $\mathrm{IL}=50$ a $400 \mathrm{gr}$ \\
\hline Retención de agua & 30 a $35 \%$ & 55 a $70 \%$ \\
\hline
\end{tabular}

El sustrato, ya sea puro o en mezclas, es el medio por el cual se sustituye el suelo para el cultivo de plantas dentro de algún contenedor. El sustrato óptimo será definido por el tipo de especie vegetal a cultivar, el tamaño del contenedor, las condiciones climáticas de la zona, el tamaño del área donde se producirá y el costo del sustrato mismo (Soria, 2012).

TABLA IV: RANGOS ÓPTIMOS PARA EL CULTIVO DE LA ESPINACA

\begin{tabular}{ccc}
\hline Variable & Mínimo & Máximo \\
\hline Temperatura & $5^{\circ} \mathrm{C}$ & $15^{\circ} \mathrm{C}$ \\
\hline $\mathrm{PH}$ & 6.5 & 7 \\
\hline Humedad & $60 \%$ & $90 \%$ \\
\hline
\end{tabular}


En esta tabla podemos observar los diferentes rangos óptimos tanto de temperatura, humedad y $\mathrm{pH}$ a los cuales se tiene que someter la espinaca para su crecimiento adecuado.

inemadere

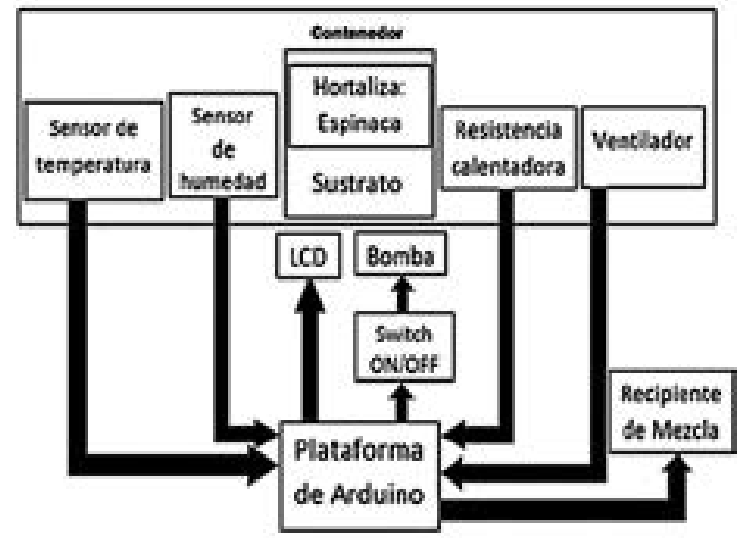

Fig. 1. Diagrama de bloques del sistema.

\section{Desarrollo}

Para controlar nuestro sistema hidropónico tenemos las siguientes variables físicas:

- Temperatura

- Humedad

$-\mathrm{pH}$ con nutrientes

Para la temperatura, el sensor a usar es un LM35. Por su resolución de 0,5 grados, es un dispositivo económico; además, cumple con los rangos de medición de temperatura que se van a manejar en el cultivo hidropónico (Daravińa, 2014). Por otro lado, se usará el sensor de humedad relativa DHT11 porque sus características, como bajo costo, disponibilidad del dispositivo y linealidad, lo perfilaron como el que mejor cubrirá las necesidades del invernadero que se elaborará (Daraviña, 2014). El acondicionamiento de nuestros sensores se realizará por medio del puente de wheatstone, que es usado comúnmente en sensores resistivos.

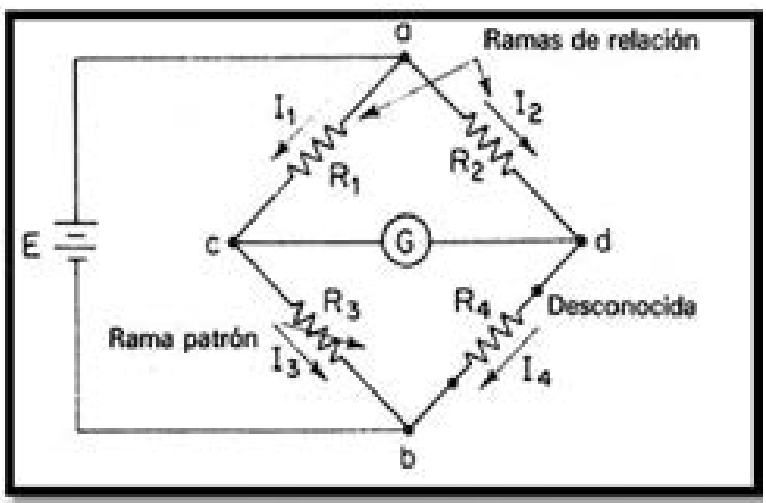

Fig. 2. Puente con sensor resistivo variable. 
El balance del puente se consigue con:

$$
\frac{\mathrm{R} 1}{\mathrm{R} 3}=\frac{\mathrm{R} 2}{\mathrm{R} 4}
$$

En esta ecuación, R3 es el valor del sensor resistivo.

El $\mathbf{~ p H}$ es un factor importante, pues de este depende el aprovechamiento de los nutrientes. Se escogió el diseńo de un pH-metro para su monitoreo (García Torres, 1991).

\section{A. Diseño}

El diseño de nuestro proyecto se centra en combinar la técnica NFT con el invernadero que recubra las condiciones necesarias. En ese sentido, este sistema cumple con la distribución de nutrientes y aclimatación (FAO, 1971). En las siguientes imágenes, se visualizan los modelos.

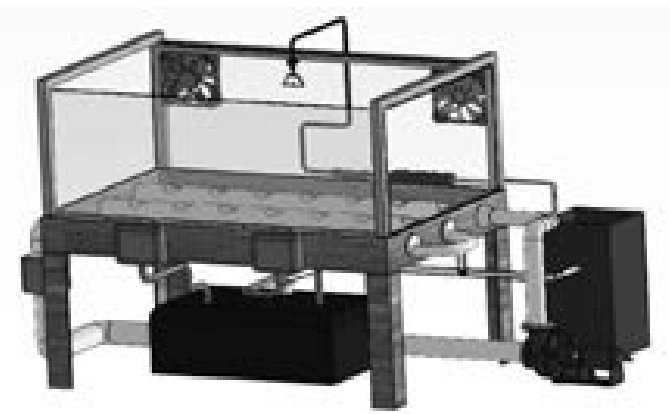

Fig. 3. Proyección de maqueta.

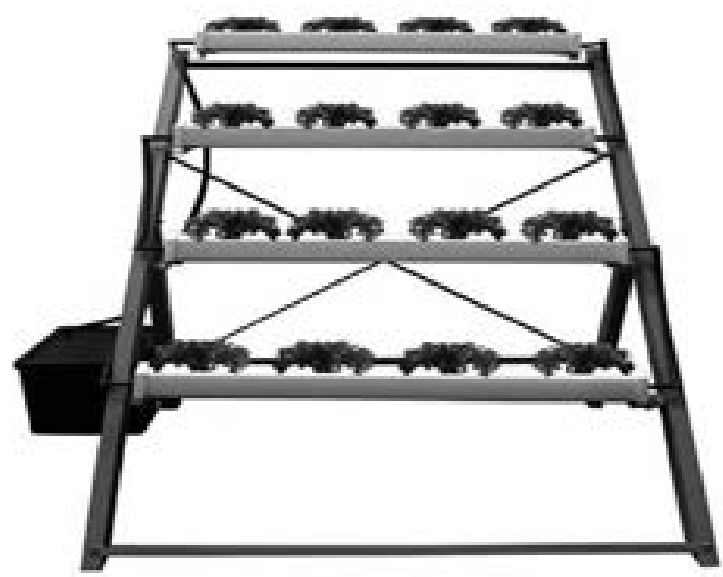

Fig. 4. Sistema NFT.

Se elaborará un sistema hidropónico en un invernadero a partir de un sistema NFT. Combinando ambos métodos, lograremos tener una mejora tanto en el riego de las plantas como en el uso de la gravedad que facilitaría llevar los nutrientes en bajada, lo que también supondría un método económico respecto a la figura 2 .

La automatización propiamente dicha se llevará a cabo a través de un timer digital. Una vez conectada la bomba de agua al timer y este a la corriente eléctrica, nuestro sistema de NFT no necesitará 
tener la bomba de agua prendida todo el día, sino solamente el tiempo necesario, ya que el timer se encargará de cortar el paso de energía a la bomba de agua según la programación (Resh, 2001).

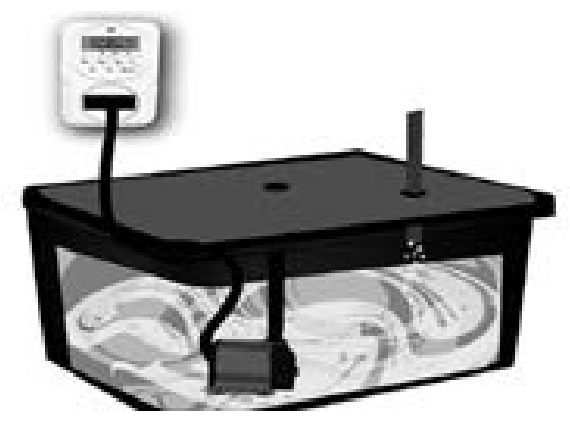

Fig. 5. Conexión timer y bomba eléctrica.

La ventaja que tenemos al utilizar el timer digital es que podemos salir de casa con la plena seguridad de que nuestro sistema seguirá funcionando (Resh, 2001). Una programación en 8 tiempos, para todo un día, podría ser la siguiente:

TABLA IV: PROGRAMACIÓN DE ON/OFF DE BOMBA

\begin{tabular}{ccc}
\hline Tiempo & Encendido & Apagado \\
\hline Primer tiempo & $08: 00$ & $08: 03$ \\
\hline Segundo tiempo & $10: 00$ & $10: 03$ \\
\hline Tercer tiempo & $12: 00$ & $12: 03$ \\
\hline Cuarto tiempo & $02: 00$ & $02: 03$ \\
\hline Quinto tiempo & $04: 00$ & $04: 03$ \\
\hline Sexto tiempo & $06: 00$ & $06: 03$ \\
\hline Séptimo tiempo & $08: 00$ & $08: 03$ \\
\hline Octavo tiempo & $10: 00$ & $10: 03$
\end{tabular}

En este caso, el tiempo de actividad para circulación es de 3 minutos y el tiempo de inactividad, de 2 horas.

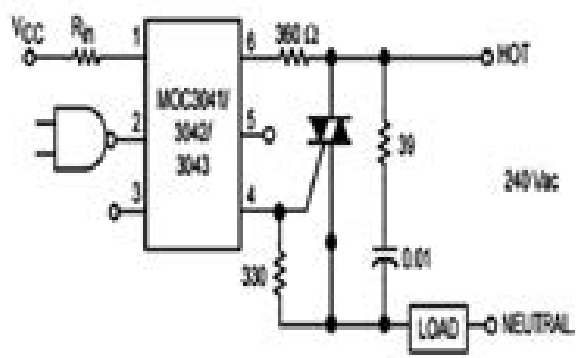

Fig. 6. Circuito de potencia para adaptar la bomba al Arduino 


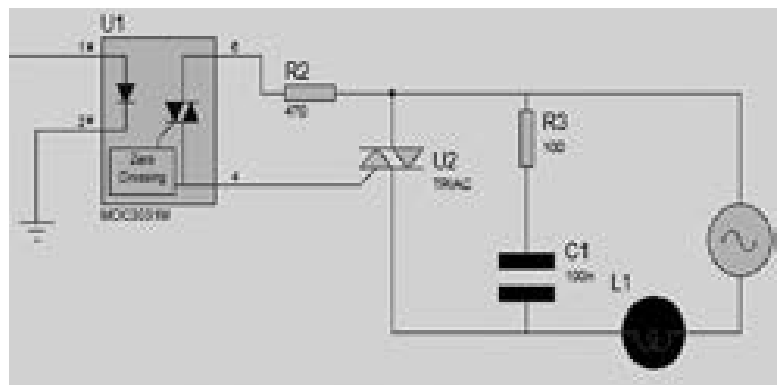

Fig. 7. Circuito de potencia en simulación.

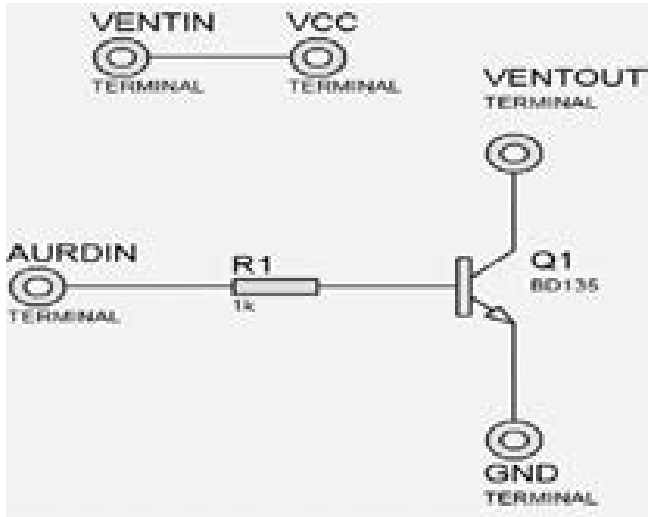

Fig. 8. Simulación del circuito para encendido y apagado del ventilador.

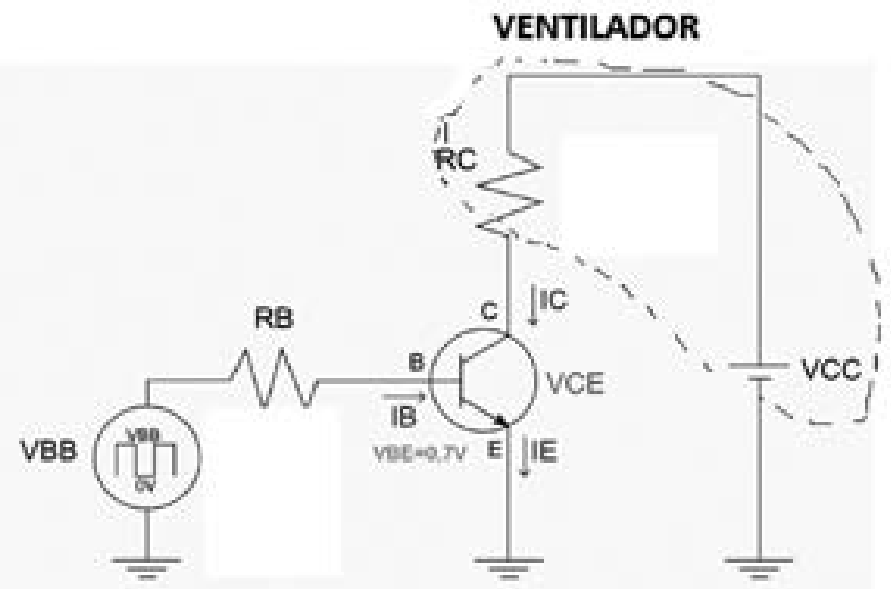

Fig. 9. Circuito con transistor BJT.

Se cumple que:

$$
\mathrm{IC}=\beta^{*} \mathrm{IB}
$$


Para que el transistor entre en corte, IC tiene que ser cero $(\mathrm{IC}=0)$, lo cual ocurre si $\mathrm{IB}=0$, y esto será cuando $\mathrm{VBB}=0$.

Además:

$$
\mathrm{VCE}=\mathrm{VCC}-\mathrm{RC}^{*} \mathrm{IC}
$$

Cuando IC $=0$, la VCE corte $=$ VCC, es decir, que en el corte a través de RC no hay corriente, y toda la tensión VCC caerá sobre el colector emisor.

Para que el transistor entre en saturación, la $\mathrm{VCE}=0$. En teoría, entre el colector y el emisor habrá un cortocircuito, que generará la corriente de colector de saturación:

$$
\mathrm{ICsat}=\mathrm{VCC} / \mathrm{RC}
$$

Corriente que la carga necesita para funcionar adecuadamente, con la corriente que necesita RC, a partir de IC $=\beta^{*}$ IB se puede obtener el valor de la corriente de la base necesaria para obtener la corriente de saturación, cuya fórmula es:

$$
\mathrm{IB}=\mathrm{ICsat} / \beta
$$

De esta forma, se logra asegurar la saturación, ya que si $\beta$ cambia, IB también cambiará. Pero ICsat no se verá afectada.

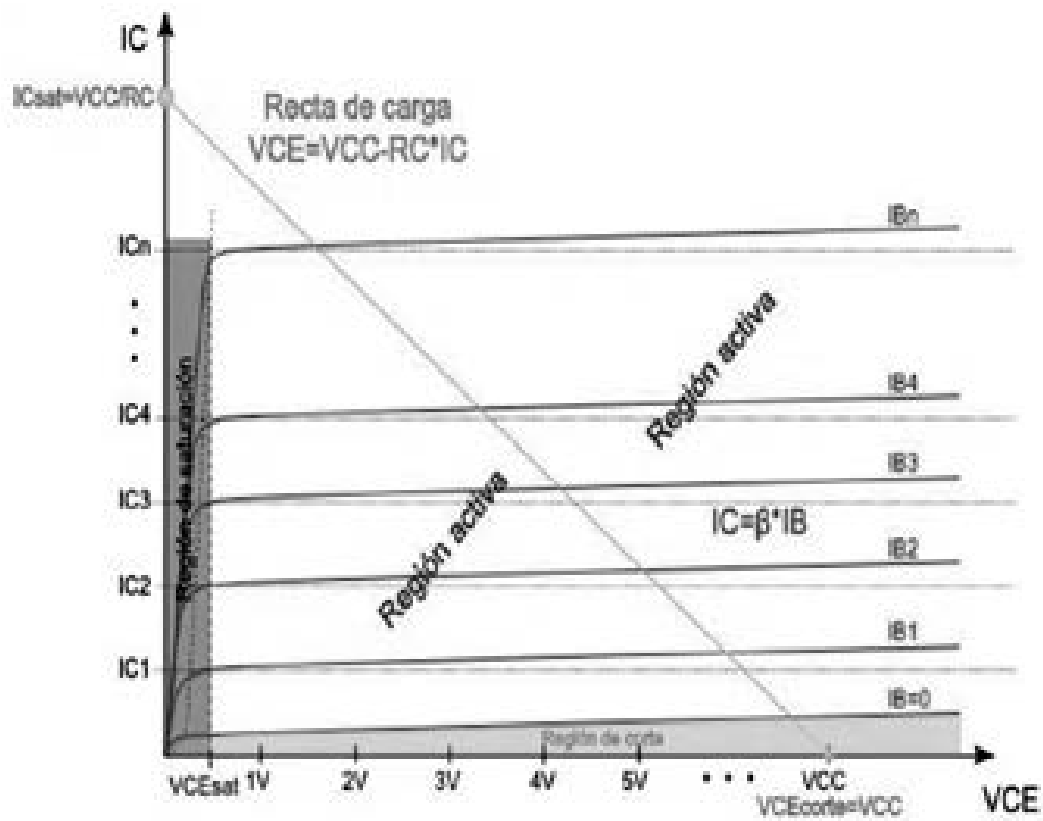

Fig. 10. Gráfica del transistor BJT; corte, saturación y punto de operación $Q$.

Sistema de calentamiento en el ambiente por medio de un alambre de nicrom 


\section{TABLA V. ESPECIFICACIONES DEL SENSOR DHT11}

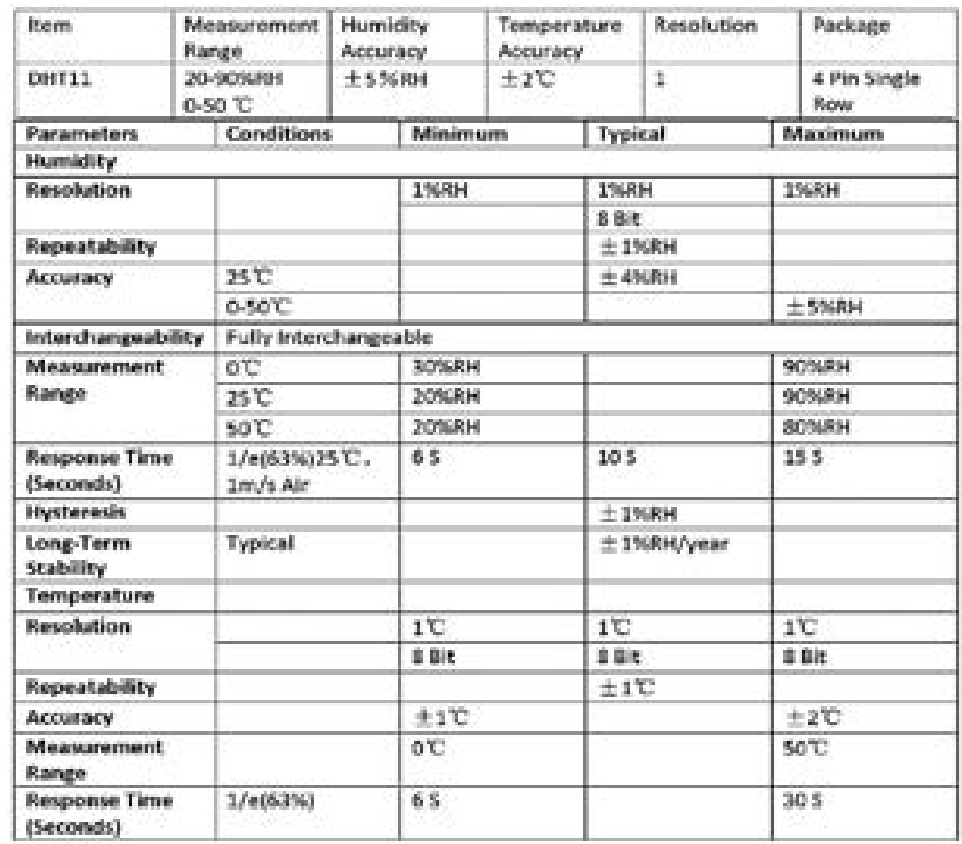

\section{B. Programación}

Diagrama de flujo para la programación del Arduino en el funcionamiento del sistema para el control de las variables ambientales

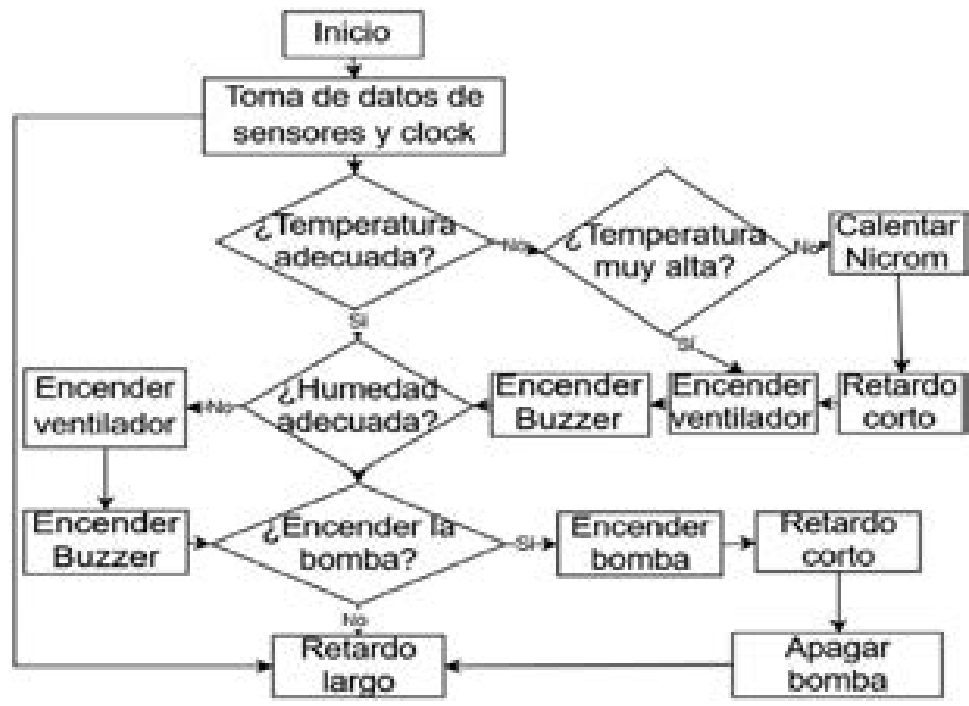




\section{Pruebas}

Inicialmente, se realizaron simulaciones de los diversos sectores del sistema para comprobar su funcionamiento individual. El circuito de potencia resulta típico si se acopla un optoacoplador y un triac. Dado que el simulador no reconoce una bomba de agua, se probó su funcionamiento con una lámpara que, en esencia, recibe la misma configuración.

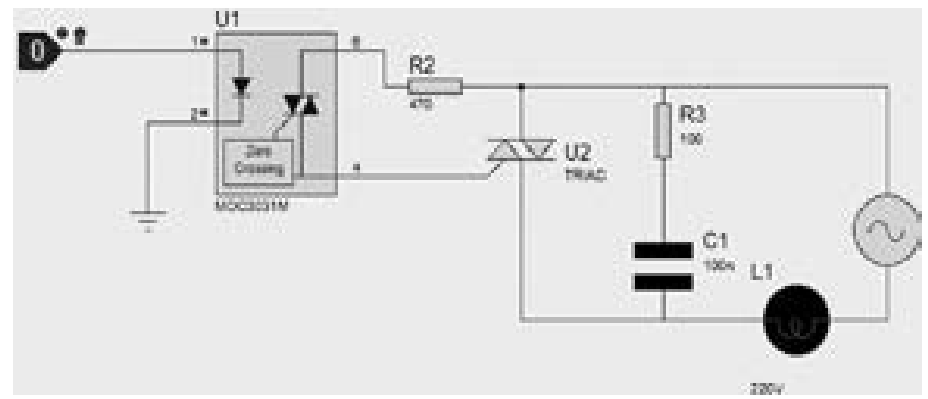

Fig. 11. Simulación del circuito de potencia para 'O' lógico.

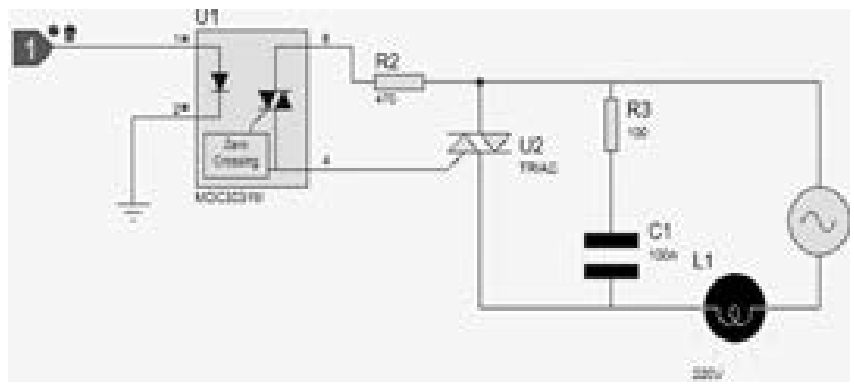

Fig. 12. Simulación del circuito de potencia para '1' lógico.

Para realizar las pruebas, y con el fin de simular las distintas condiciones climáticas y ambientales a las que puede ser expuesto el sistema, decidimos someterlo a distintos cambios de temperatura y humedad. Se comprobó, entonces, que en cada caso el sistema mismo estabilizó las condiciones internas para las que fue programado, las mismas que se consideraron necesarias para la supervivencia y crecimiento óptimo de la espinaca.

A continuación, se muestra el crecimiento de la hortaliza semana tras semana, junto con el display que señala las condiciones del ambiente.

\section{RESULTADOS}

Nuestro cultivo logró crecer y obtuvimos los resultados que esperábamos; por lo tanto, el ambiente creado para su supervivencia fue el más propicio.

La pantalla LCD, por medio del procesamiento del Arduino, muestra continuamente las variables a controlar y nos permite la visualización del proceso.

Las hortalizas tienen la propiedad de crecer en menos tiempo que otras plantas. Esto se comprobó al realizar el proyecto, pues pudimos observar el producto en crecimiento hasta un poco antes del final. 


\section{CONCLUSIONES}

El control automático nos permitió optimizar de manera asombrosa la producción de la espinaca como hortaliza, lo que ahorró la mitad del tiempo. Asimismo, no fue necesario un espacio abundante ni dedicación constante, pues el sistema era autónomo y fue capaz de mantenerse en condiciones ambientales favorables.

La plataforma Arduino fue la interfaz que permitió el control de variables. Esta resultó fácil y práctica de programar; de esta forma, fue muy útil en el proyecto.

Las hortalizas son vegetales cuyo ambiente favorable para su crecimiento es fácil de simular a comparación de otros productos que requieren de variables como la altitud.

\section{OBSERVACIONES}

Se logra gran control y optimización del agua y de nutrientes, ya que no hay pérdida por drenaje, evaporación o filtraciones; solo se pierde el agua transpirada por la planta. Como los volúmenes son reducidos, es más fácil desinfectar la solución nutritiva para el control o prevención de enfermedades antes de volverla a usar.

Se logra un sistema radicular pequeño pero que puede soportar a una planta grande. Las plantas cosechadas se remueven fácilmente.

Es indispensable no excederse en las cantidades recomendadas, pues podrían producirse intoxicaciones en los cultivos (Marulanda, 2003).

\section{RECOMENDACIONES}

Estos nutrientes, bien sea que vengan en forma de polvo o de líquido, se deben aplicar en el área de las raíces, tratando de mojar lo menos posible sus hojas para evitar toxicidad o la aparición de enfermedades.

No se deben confundir los nutrientes para uso hidropónico con los nutrientes foliares (Marulanda, 2003).

\section{REFERENCIAS}

[1] Alpízar Antillón, L. (2004). Hidroponia: cultivo sin tierra. Elementos básicos para desarrollar la técnica simple de cultivar plantas sin tierra en pequeños espacios. Costa Rica: Cartago Editorial Tecnológica.

[2] Basterrechea, M. (febrero, 2016). «Hidroponía casera». Recuperado de http://www.hidroponiacasera.net/.

[3] Castañeda, F., De Valverde, Ch., y García, L. (abril, 1997). Manual de cultivos hidropónicos populares: producción de verduras sin usar tierra. Guatemala: INCAP, 55 (3), pp. 1-6.

[4] Daraviña Peña, G. y Valencia Guitierrez, R. (2014). Diseño de sistema se control de optimización ambiental para cultivo hidropónico (Tesis para optar el título de Tecnólogo en Mecatrónica) Universidad Tecnológica de Pereira.

[5] FAO (1971). «Necesidades de Energía y Proteínas». En Alimentación y nutrición N7. 
[6] García Torres, A. (1991). Medidor de pH de bajo costo. Educación química, 2 (2). Recuperado de file://C:/Users/Maricarmen/Downloads/pdf87.pdf, pp. 81-85.

[7] Guzmán Díaz, G. (2004). Hidroponía en Casa: una actividad familiar. San José, Costa Rica: Ministerio de Agricultura y ganadería-Sistema Unificado de Información Institucional.

[8] Marulanda C., e Izquierdo J. (2003). La huerta hidropónica popular (3a ed.). Santiago de Chile: Oficina Regional de la FAO para América Latina y el Caribe.

[9] Murray, R. et. al. (1993). Bioquímica de Harper (12a ed). Bogotá: El Manual Moderno.

[10] Resh, H. M. (2001). Cultivos hidropónicos: nuevas técnicas de producción. Madrid: Mundi-Prensa.

[11] Sampero Ruiz, G. (1999a). Hidroponía comercial. México D. F.: Editorial Diana.

[12] Sampero Ruiz, G. (1999b). Hidroponia básica: el cultivo fácil y rentable de plantas sin tierra. México D. F.: Editorial Diana.

[13] Soria Campos, J (2012). $6^{\circ}$ Curso de hidroponía básica para principiantes. Recuperado de http://www. asohofrucol.com.co/archivos/biblioteca/biblioteca_247_Curso\%20Hidropon\%C3\%ADa\%20 Basica.pdf.

[14] Texier, W. (2013). Hidroponía para todos: todo sobre la horticultura en casa. Varela, F. (Trad.). París: Mama Editions.

[15] Via, J., Elvira, V., Santamaria I. y Eickhoff, R. (2009). "Analog Antenna Combining for Maximum Capacity under OFDM Transmissions». International Conference on Communications (ICC 2009), Dresden-Alemania.

\section{ENLACES DE INTERÉS}

Manual de Hidroponía. Disponible en http://www.oasisfloral.mx/pdf/manualhidroponia.pdf.

Información sobre invernaderos. Disponible en http://losinvernaderos.blogspot.pe/2010/10/sistemas-de-riego-en-cultivos.html.

N. T. F. (Nutrient film technique) y su instalación. Disponible en http://hydroenv.com.mx/catalogo/ index.php?main_page=page\&id=102.

Sunrom technologies. Disponible en http://robocraft.ru/files/datasheet/DHT11.pdf. 